\title{
Florula Tsusimensis
}

Auctore

Y. Yabe.

\section{HYMENOPHYLLACEAE.}

Trichomanes parvulum Poiret. Blume Enum. Pl. Jav. Fil. p. 223 ; Hook. Sp. Fil. I. p. 118 t. 89 A ; Miq. Prol. Fl. Jap. p. 347 ; Bak. et Hook. Syn. Fil. ed. II. p. 75 ; Fr. et Sav. Enum. Pl. Jap. II. p. 207 ; Christ Farnkr. d. Erd. p. 27 ; Yabe. Fil. Kor. Uchiy. in But. Mag. Tokyo. XVII. p. 63.

Hab. circa. Izuhara.

Tri. filicula Bory. Bak. et Hk. Syn. Fil. p. 81 ; Fr. et Sav. Enum. Pl. Jap. II. p. 207 ; Makino. Phan. Pter. Jap. Ic. Ill. pl. XV.

Didimoglossum filicula Desv. Mem. Soc. Linn. VI. p. 381 ; V. D. Bosch. Hym. Jav. p. 35 t. 16 ; Miq. Cat. Mus. Lugd. Batav. p. 129.

Hab. Izuhara (ipse).

Hymenophyllum barbatum Miq. Prol. Fl. Jap. p. 3 ; Bak. Syn. Fil. p. 68 ; Fr. et Sav. Enum. Pl. Jap. II. p. 206 ; Sadebeck in Engl. et Prantl. Nat. Pf. Fam. I. abt. 4. p. 110.

Hab. Ins. Tsusima (Wilford, 846); in monte Yatateyama (ipse); in monte Shiratake (K. Hirata 187).

H. polyanthos Sm. Hk. Sp. Fil. I. p. 106 ; Bak. Syn. Fil. p. 60 ; Fr. et. Sav. Enum. Pl. Jap. II. p. 226 ; Christ Farnkr. p. 16.

Hab. 'Tsushima (sec. Baker 1. c.)

\section{Polypodiaceæ.}

Struthiopteris germanica Willd. Sp. Pl. V. 1. 288 ; Christ, Farnkr. 1. 285 ; Diels in Nat. Pfl. Fam. I. 4. p. 165 ; Yabe, 1. c. 64.

Onoclea germanica (Willd) Hk. Sp. Fil. IV. p. 161 ; Miq. Prol. 345 ; Baker, Syn. Fil. p. 46 ; Fr. et Sav. Enum. II. p. 204.

Hab. Izuhara (ipse).

St. orientalis Hook. 2nd. Cent. Fern, t. 4 ; Christ, Farnkr. p. 285; Diels in Nat. Pf. Fam. I. 4. p ; Palibin. Consp. Fl. Kor. III. 39 ; Yabe 1. c. 64.

Onoclea orientalis Hk. Sp. Fil. IV. p. 161 ; Miq. Prol. p. 343 ; Bak. Syn. p.• 46 ; Fr. et Sav. Enum. II. p. 204.

Hab. in. monte Yatateyama (ipse).

Nephrodium decipiens Hk. Sp. Fil. IV. p. 86. t. 243 ; Bak. Syn. p. 260. 
Hab. in monte Siratake (K. Hirata. 181).

N. gracilescens Hk. var. glanduligerum Baker, Syn. Fil. p. 262.

Hab. circa Izuhara (ipse et K. Hirata).

N. Thelypteris Desv. Hook. Sp. Fil. IV. p. 88 ; Bak. Syn. p. 271.

Aspidium Thelypteris Sw. Syn. Fil. p. 57 ; Fr. et Sav. Enum. II. p. 237.

Hab. Izuhara (ipse)

N. lacerum Bak. Syn. Fil. p. 273; Diels in Nat Pll. Fam. I. 4. p.

Aspidium lacerum Sw. Syn. Fil. p. 55; Kunze, Pterid. Jap. p. 572 ; Miq. Prol. p. 341 ; Fr. et Sav. Enum. II. p. 238.

Hab. Tsusima (sec. Bak. l. c.), Izuhara (ipse).

N. erythrosorum Hk. Sp. Fil. IV. p. 120. t. 253; Bak. Syn. p. 273; Diels in Nat. Pff. Fam. I. 4. p. 173.

Aspidium erythrosorum Eaton, in A. Gray Pl. Jap. p. 330 ; Miq. Prol. p. 342 ; Fr. et Sav. p. 239; Christ, in Warb. Monsunia. I. 80.

A. filix-mas Sw. var. erythrosorum Christ, Bull. L'herb. Bois. Tome. VII. n. 11. 1899. p. 821.

Hab. Izuhara et in monte Yatateyana (ipse), (Warburg).

N. Totta Diels in Nat. Pfl. Fam. I. 4 p. 170, fig. $91 \mathrm{G}$; Gymnogramme Totta Schlecht. Hk. Sp. Fil. V. 138 ; Bak. Syn. p. 376 ; Fr. et Sav. Enum. II. p. 247.

Hab. Tsusima (Wilford. sec. Hk. l. c.) ; Kamoize (ipse).

N. sophoroides Desv. Ann. Soc. Linn. VI. p. 256 ; Bak. Syn. p. 289 ; Diels in Nat. Pfl. Fam. I. 4. p. 178.

Aspidium sophoroides Sw. Syn. Fil. p. 48 ; Kunze, Pter. Jap. p. 555; Miq. Prol. p. 342 ; Fr. et Sav. Enum. II. p. 242.

Hab. Izuhara (ipse).

Polystichum lepidocaulon J. Sm. Diels in Nat. Pfl. Fam. I. 4. p. 190; Palibin l. c. XI. 41.

Aspidium lepidocaulon H1k. Sp. Fil. IV. p. 12, t. 217; Miq. Prol. p. 340 ; Bak. Syn. p. 250 ; Fr. et Sav. Enum. Pl. Jap. II. p. 230.

Hab. Tsushima (Wilford! sec. Hk. 1. c.); per totam insulam (ipse).

P. craspedosorum Diels in Nat. Pfl. Fam. I. 4. p. 189.

Aspidium craspedosorum Maxim. Mél. Biol. VII. p. 341 ; Bak. Syn. Fil. p. 492 ; Fr. et Sav. l. c. p. 231 ; Makino in Phaner Pter. Jap. Ic. Mll. no. 9. pl. 44.

Hab. in radice montis Mitake (ipse).

P. tripteron J. Sm. Diels in Nat. Pf. Fam. I. 4. p. 91. fig. 99. G. H.

Aspidium tripteron Kze. Pter. Jap. in Bot. Zeit. VI. p. 509 ; Hk. 2nd Cent. Fern, t. 56 ; Sp. Fil. IV. p. 15. ; Miq. Prol. p. 340 ; Bak. Syn. p. 254 ; Fr. et Sav. Enum. II. p. 232.

Hab. 'Tsusima (Wilford! sec. Hk. l. c.) ; Kurochozaka (ipse) ; in- moute Siratake (K. Hirata). 
P. aculeatum Roth. var. japonicum Christ, Extrait Bull. Soc. Bot. Suisse, 1893. p. 13 ; Yabe 1. c. 65.

Aspidium aculeatum Doll. var. japonicum Fr. et Sav. Enum. II. p. 231.

Polystichum japonicum Diels. in Nat. Pfl. Fan. I. 4 p. 191.

Hab. in montes Mitake et Yatateyama (ipse).

P. tsus-simense (Hk.) Diels. Nat. Pfl. Fam. I. 4. p. 191.

Aspidium aculeatum Sw. forma. tsus-simense Bak. Syn. p. 252.

Asp. tsus-simense Hk. Sp. Fil. IV. p. 16, t. 220; Miq. Prol. p. 340; Fr. ct Sav. Enum. p. 231.

Hab. Tsusima (Wilford! see. Hk. l. c.)

P. aristatum Prol. Tentam. Pter. p. 83 ; Diels in Nat. Pfl. Fam. I. 4. p. 193. Aspidium aristatum Sw. Syn. Fil. p. 53 ; Schk. Fil. p. 44 t. 42 ; Hk. Sp. Fil. IV. p. 28 ; Miq. Prol. p. 341 ; Bak. Syn. p. 255 ; Fr. et Sav. Enum. II. p. 234 ; Christ. Bull. L'herb. Buis. 1902. p. 828.

Hab. Tsusima (Wilford! sec. Ilis. 1. c. Faurie sec. Christ.); per totam insulam (ipse).

P. varium Presl. Diels in Nat. I’fl. Fam. I. 4. p. 193 ; Palibin 1. c. III. p. 41 ; Yabe 1. c. 65.

Aspidium variım Sw. Syn. Fil. p. 51 ; Kze. Bot. Zeit. VI. p. 571 ; Hk. Sp. Fil. IV. p. 20 t. 226 ; Miq. Prol. p. 342 ; Bak. Syn. 254 ; Fr. et Silv. Enum. II. p. 233.

IIal). Izuhara (K. Hirata).

P. falcatum (I) Dicls, Nat. P'f. Fam. I. 4. p. 194 ; Yabe, 1. c. 65.

Aspidium falcatum Sw. Syn. Fil. 1. 43; Hook. et Arnot. Bot. of Beech. Voy. p. 274 ; Kze. Pter. Jap. p. 558 ; Ilk. Fil. Wxot. t. 92 ; Hk. Sp. Fil. IV. p. 40 ; Bak. Syn. Fil. p. 257 ; Miq. Prol. p. 340 ; Fr. et Sav. Enum. II. p. 234; Christ in Warb. Monsunia I. 76.

Hab. Per totam insulam (ipse). (Warburg).

var. Fortunei (Bak) Syn. Fil. p. 257.

Cyrtomium Fortunei J. Sm. Fern Br. Foreign. 1866.

Hab. Izuhara (ipse).

Davaliia bullata Wallich. ILook. Sp. Fil. I. p. 169 t. 50 . b; Mic. Prol. p. 34t ; Bak. Syn. Fil. p. 97 ; Fr. et Sav. II. j. 208 ; Christ Farnkr. p. 301.

Hab. Oyamadake (ipse)

Microlepia Wilfordii Moore. Davalia IVilfordii Bak. Syn. p. 98; Fr. et Sav. Enum. II. p. 208.

Hab. Izuhara.

M. hirsuta (Sw.) Prosl.

Davallia hirsuta Sio. Syn. Fil. 131, 343; Kzo. Pler. Jap. p. 541 ; Bak. Syn. p. 98 ; Fr. et Siv. Enum. H. p. 209.

D. pilosella Hk. 2nd. Cent. Fern, 96. 
Hab. 'Tsusima (sec. Hk. l. c.)

M. strigosà Presl.

Davallia strigosa Sw. Kunze, Pter. Jap. p. $5+2$; Miq. Prol. p. 344; Bak. Syn. p. 98 ; Fr. ct Sav. Enum. II. p. 210.

Hab. 'Takesiki (ipse).

M. marginalis Hance. Bedd. Handb. F. Br. Ind. p. 64; Christ, Farnkr. p. 307 ; Diels, in Nat. Pfl. Fam. I. 4. p. 215.

Davallia marginalis Bak. et Hk. Syn. Fil. p. 98 ; Fr. et Sav. Enum. II. p. 209.

Hab. circa Izuhara (ipse).

Odontosoria chinensis J. Sm. Diels in Nat. Pfl. Fam. I. 4. p. 215.

Lindsaya tenuifolia Mctt. Ann. So. Nat. Par. ser. 4. XV. p. 64 ; Christ, Farnkr. p. 296.

Davallia tenuifolia Sw. Syn. Fil. 1. 133 ; Miq. Prol. p. 344 ; Fr. et Sav. Enum. II. p. 210 ; Bak. Syn. Fil. p. 102.

Hab. circa Izuhara. (ipse).

Athyrium yokoscence (Fr. et. Sav). Christ. Bull. Herb. Bois. 1896. p. 668 ; Yabe. 1. c. 66 .

Asplenium yokoscence Fr. et Sav. Enum. Pl. Jap. II. p. 622.

Hab. Tsushima, circa Izuhara (ipse).

Diplazium lanceum Presl. Berld. Handb. F. Br. Ind. p. 174; Christ, Farnkr. p. 215 ; Diels, Nat. Pfl. Fam. I. 4. p. 225.

Asplenium lanceum Thunb. Fl. Jap. p. 333 ; Ic. Pl. Jap. II. t. 18 ; Sw. Syn. Fil. p. 74 ; Hook. Sp. Fil. III. p. 235 ; Bak. Syn. p. 228 ; Fr. et Sav. Enum. II. p.

Hab. T'susima (Wilford, sec. Hk. l. c.) ; valde commune (ipse)!

D. japonicum Bedd. Handb. F. Br. Ind. p. 180 ; Christ, Bull. L'herb. Boiss. Tome IV. no. 10. 1896. p. 669 ; Diels in Nat. Pfl. Fam. I. 4. p. 226.

Asplenium Japonicum Thunb. Fl. Jap. p. 334; Bak. Syn. p. 234 ; Fr. et Sav. Enum. II. p. 227.

Hab. circa Izuhara (ipse).

Scolopendrium sibiricum Hk. Sp. Fil. IV. p. 4 ; 2nd Cent. F. t. 35 ; Bak. Syn. p. 248 ; Christ, Farnkr. p. 213 ; Diels in Nat. P'f. Fam. I. 4. p. 231.

Hab. Tsusima (Wiliord n. 790. sec. Hk. l. c.).

Asplenium furcatum Thumb. Hk. Sp. Fil. III. p. 166.

Hab. Tsusima (Wright, see. Hk.)

A. incisum Thunb. Trans. Linn. Soc. II. p. 342 ; Kze, Pter. Jap. p. 523 ; Miq. Prol. p. 337 ; Bak. Syn. p. 217 ; Fr. et Sav. Enum. II. p. 221 ; Diels in Nat. Pfl. Fam. I. 4. p. 241.

Hab. Tsusima (Wilford n. 753. sec. Hk. 1. c.) ; circa Izuhara (ipse.)

A. Wardii Hk. 2nd Cent. F. t. 33 ; Sp. Fil. IIl. p. 189 ; Miq. Prol. p. 337 ; Bak. Syn. p. 217 ; Fr. et Sav. Enum. II. p. 222 ; Palibin l.c. III. 42.

Hab. Tsusima (IVilford, no. 717. sec Hk. l. c.) 
A. Dayallioides Hk. Sp. Fil. III. p. 212 ; 2nd. Cent. F. t. 40 ; Bak. Syn. p. 222 ; Fr. et Sav. Enum. II. p. 222.

Hab. Tsusima (Wilford, no. 791 sec Hk. l.c.); Kamoize (ipse).

A. rutaefolium Kze. Limnea X. p. 521 ; Presl. Tent. Pter. p. 108 ; Hk. Sp. Fil. III. p. 206 ; Bak. Syn. 222; Fr. et Sav. Enum. II. p. 222 ; Diels Nat. Pfl. Fam. I. 4. p. 242 ; Makiuo, Phin. Jap. Ic. ill. II. n. 1. t. 65.

Hab. Tsusima (Wilford, sec. Hk. 2nd Cent. l.e.), in monte Siratake (K. Hirata 179.)

A. abbreviatum Makino. Pl. Jap. nor. r. min. cogn. in Bot. Mag. Tokyo, XIII. p. 12 ; Id. Ph. Pter. Jap. Jcon. Ill. II. n. 1. t. 63.

Hab. Tsusima (ipse)

A. Saulii Hook. Christ in Warb. Monsunia I. p. 72.

Hab. Ins. Tsusima (Warburg).

Coniogramme fraxinea Fee. Diels in Nat. Pfl. Fam. I. 4. p. 262 ; Yalbe. 1. c. 67.

Gymnogramme javanica Blume, Enum. Pl. Jav. Fil. p. 112; Fl. Jav. Fil. p. 95. t. 41 ; Hk. Sp. Fil. p. 145 ; Miq. P'rol. p. 335 ; Bak. Syn. Fil. p. 381 ; Fr. et Sav. Enum. II. p. $2+8$.

Hab. Izuhara (ipse).

C. japonica Dicls, Nat. Pfl. Fam. I. 4. p. 262.

Gymnogramme japonica Desv. Hk. Sp. Fil. V. p. 151 ; Miq. Prol. p. 335 ; Kze. Pter. Jap. p. 493 ; Bak. Syn. p. 390 ; Fr. et Sar. Enum. p. 249.

Hab. Izuhara (ipse).

Cryptogramire japonicum Prantl. Diels, Nat. Pfl. Fam. I. 4. p. 279. Ony. chinm japonimm Kze. Pteria. Jap. p. 507 ; Bak. Syn. p. 143 ; Fr. et. Sav. Enum. 1. 212 .

Hab. Per totam insulam, in sepibus arenosisve (ipse).

Plagiogyra adnata Bedd. Fern Br. Ind. t. 51 ; Handb. F. Br. Ind. p. 127 ; Diels, in Nat. Pfl. Fam. I. 4. p. 282 ; Fl. C'ent. Chin. p. 200.

Lomaria adnata. Bl. En. Fil. Jav. p. 205; Hk. Sp. Fil. III. p. 19 ; Bak. Syn. p. 182 ; C. B. Clark. Rev. F. Br. Ind. p. 472.

Hab. circa Izuhara (K. Hirata 157).

Adiantum monochlamys Eat. Proc. Am. Acar. IV. p. 110 ; Hk. 2nd. Cent. F. t. 50. Mic. Prol. 385; Bak. Syn. p. 125 ! Fr. et. Sav. p. 211 ; Christ, Farnkr. p. 140 ; Diels Nat. Pfl. Fam. I. 4. p. 284.

Hab. Tsusima (Wilford, sec. Baker l.e.); in montibus borealis (Mitáke, Kuro(chozaka, etc. ipse).

Pteris cretica L. Sw. Syn. p. 96; Thumb. Fl. Jap. p. 332 ; Kunze. Pt. Jap. p. 538 ; Hk. Sp. Fil. II. p. 160 ; A. Gray, Pl. Jap. p. 329 ; Miq. Prol. p. 336 ; Bak. Syn. Fil. p. 154; Fr. et Sav. Fnum. II. p. 212; Christ Farukr. p. 165; Diele in Nat Pfl, Faum. I, 4. p. 292. 
Hab. Izuhara (ipse).

P. serrulata L. f. Suppl. p. 425 ; Kunze, Pter. Jap. p. 539 ; Miq. Prol. p. 336; Bak. Syn p. 155 ; Fr. et Sav. Enum. II. p. 215 ; Christ, Farnkr. p. 166 ; Diels in Nat. Pfl. Fam. I. 4. p. 292 ; Makino, Phan. Pter. Jap. Icon. Ill. I. n. 9. t 45.

Hab. Izuhara (ipse)

P. semipinnata L yar. dispar Bak. Syn. p. 157 ; Christ. Farnkr. p. 166.

Pt. dispar Kunze. Pter. Jap. p. 539.

Hab. in monte Tatera yama (ipse).

Pteridium aquilinum Kuhn. “ Botanik v. Ost. Afrik. in v. d. Decken Reisen III. 3.11 Palibin, Cousp. Fl. Kor. III. 42 ; Yabe. 1. c. 67.

Pteris aquitina I. Sp. 1533 ; Thunb. Fl. Jàp. p. 332 ; Kunze Pter. Jap. p. 540 ; Miq. Prol. p. 336 ; Bak. Syn. p. 162 ; Fr. et Sav. Enum. II. p. 215 ; Christ, Farnkr. p. 164.

Hab. Per totam insulam (ipse).

Vittaria japonica Miq. Prol. p. 333.

V. lanceola Christ. Bull. L'herb. Bois. Tome IV. N. 10. 1896. p. 674.

Hab. Izuhara (ipse)

Drymoglossum carnosum Hook. Gen. Fil. t. 78. A ; Sp. Fil. V. p. 189 ; Bak. Syn. p. 397 ; Fr. et Sav. Enum. II. p. 250 ; Christ. Farnkr. p. 131 ; Luerss. in Engl. Bot. jahrb. IV. p. 364 ; Yabe, 1. c. 67.

D. subcordatum Fée. Gen. Fil. p. 94. t. 9. A. f. 1 ; Christ. in Warb. Monsunia. I. 66 .

Hab. Per totam Insulam. (ipse. Warburg sec Christ. 1.c.)

Polypodium (Pleopetlis) superficiale Blume. Fil. Jav. p. 136. t. 56. f. 1 ; Hk. Sp. Fil. V. p. 71 ; Bak. Syn. p. 356 ; Fr. et Sav. Enum. II. p. 246 ; Diels in Nat. Pfl. Fam. I. 4. p. 315 ; Fl. C. China. p. 203 ; Christ. Bull. Herb. Bois. 1902. p. 831.

Hab. 'Tsusima (Wilford, sec. Hk. l.c., Faurie sec. Christ), in monte Yatateyama Izuhara. (ipse).

P. ensatum Thunb. Trans. Limn. Soc. II. p. 341 ; Kunze, Pter. Jap. p. 494 ; Hk. Sp. Fil. V. p. 72 ; Bak. Syn. 361 ; Fr. et Sav. Enum. II. p. 246 ; Diels Nat. Pfl. Fam. I. 4. p. 315.

Hab. Izuhara, Yatateyama (ipse)

P. phymatodes L. Bak. Syn. p. 365 ; Fr. et Sav. Enum. II. p. 247.

Hab. Tsusima (sec. Bak. l.c.).

P. lineare Thumb. Fl. Jap. p. 335 ; Asa Gray, Pl. Jap. p. 329 ; Miq. Prol. p. 334 ; Bak. Syn. p. 354 ; Fr. et Sav. Enum. II. 245 ; Christ, Farnkr. p. 102 ; Diels in Nat. Pfl. Fam. I. 4. p. 315 ; T. Makino, Phaner. Pter. Jap. Ic. Ill ; Christ. in Monsunia I. 61.

Hab. circa Izuhara (ipse et. K. Hirata., Warburg.)

yar. Onoei (Fr. et Sav.) Makino. 